\title{
Technical Features and Research Progress of Separating Impurities in Producing Tantalum (Niobium) Oxide by Traditional Technology
}

\author{
Huijuan Yan $^{1}$, Desheng Tang ${ }^{2}$ \\ ${ }^{1}$ Jiujiang Nonferrous Metallurgy Company Limited, Jiujiang, China \\ ${ }^{2}$ Jiangxi Tungsten Industry Group Company Limited, Nanchang, China
}

Email address:

349776921@qq.com (Huijuan Yan)

\section{To cite this article:}

Huijuan Yan, Desheng Tang. Technical Features and Research Progress of Separating Impurities in Producing Tantalum (Niobium) Oxide by Traditional Technology. International Journal of Mineral Processing and Extractive Metallurgy. Vol. 3, No. 2, 2018, pp. $29-36$.

doi: 10.11648/j.ijmpem.20180302.13

Received: June 21, 2018; Accepted: July 7, 2018; Published: August 14, 2018

\begin{abstract}
In order to meet the demand for high purity tantalum and niobium products and to adapt to the complexity of tantalum niobium ore and waste in smelting, it was necessary to prepare high pure tantalum (niobium) oxide by traditional technology. In this paper, the process features, principles and functions of the decomposition, extraction, neutralization, washing and calcination in the traditional technology were described in detail, and the key factors which affect the separation of impurities in each process and the influences of various factors on the process and product quality were analyzed. Extraction was the key process to remove metal impurities in products. The main factors affecting the extraction separation are acidity, grade efficiency, temperature, extractant, concentration of tantalum/niobium, mass ratio of tantalum to niobium and volume ratio. In the process of precipitation and calcination the reagent purity, the equipment material, and the environmental cleanliness must be concerned to avoid the inclusion of impurity elements. The research and progress of impurity removal technology in tantalum (niobium) oxide production process were reviewed. The innovation of the traditional technology can meet special low requirement of one or several elements in the product, such as fluorine, tungsten, antimony, etc. For the future the basic research on the existence state and transformation behavior of tantalum and niobium and other elements in each process of traditional technology should be strengthened.
\end{abstract}

Keywords: Traditional Technology, Tantalum (Niobium) Oxide, Separating Impurities, Extraction, Precipitation, Wash

\section{Introduction}

In recent years, with the reduction of tantalum/niobium mineral resources, the grade of tantalum / niobium for smelting is decreased, the specie of impurities is increased and the content is rising, the proportion of the poor ore and recycled waste material increases, which makes the smelting process control more difficult and the purity of the product is difficult to improve. The application of tantalum/niobium in the field of high technology, such as the electronics industry and superconductivity, has been expanded, and the higher requirements for the purity of tantalum (niobium) oxide which is the intermediate product of preparing tantalum (niobium), are also putted forward. Therefore, in order to adapt to the complexity of the ore source and meet the demand of industrial chain terminal products, the production conditions of tantalum /niobium products must be adjusted accordingly and the content of the impurity elements must be strictly controlled.

Tantalum (niobium) oxide is divided into industrial grade and high-purity grade according to its purity, and high-purity level requires tantalum oxide (niobium) more than $99.99 \%$. The purity of ultra high purity tantalum (niobium) oxide produced by Jiujiang nonferrous metal smelting Co., Ltd. is $99.995 \%$. The traditional technology of producing tantalum (niobium) oxide consists of two major processes, namely acid digestion-solvent extraction of tantalum /niobium, and the subsequent process for precipitation-calcination tantalum (niobium). 


\section{Acid Digestion Process}

Raw materials containing tantalum/niobium are ground and are digested with hydrofluoric acid. Then sulfuric acid is added to enhance digestion and adjust the acidity. The associated elements like iron, manganese, titanium, etc. are also dissolved. After filtering insoluble impurities like aluminum, silicon, calcium, and rare earth metals, the resulting acid aqueous solution is mixed with the organic solvent.

The action of acid digestion not only causes tantalum/niobium into aqueous solution, but also adjusts the acidity of solution suitable for extraction. The residual quantity of tantalum/ niobium can be reduced in the slag, the metal recovery and the economic benefit can be improved by controlling complete reaction and the acidity of digestion. At the same time, strengthening filtration can remove the harmful impurities such as solid particles suspended in the solution or "soluble" silicon acid, so as to prevent the emulsification of the subsequent extraction process. In this process, the behavior of impurity elements is generally not controlled. The pretreatment, such as low acid initial leaching, roasting or redox, will be selected for some special ore or the impurities not effectively removed in the subsequent process.

\section{Solvent Extraction Process}

\subsection{Process Aim}

Solvent extraction is the key process of separating tantalum and niobium from impurities. It is the most important process of tantalum/niobium hydrometallurgy. The control and operation of this process is not only directly related to the quality and cost of products, but also related to the connection of the front and back processes [1]. Due to the different climatic conditions in north and south China, two extraction systems are used for tantalum/niobium hydrometallurgy. At present, the Orient Tantalum Industry Company and the JinLian Industrial Company use $\mathrm{HF}-\mathrm{H}_{2} \mathrm{SO}_{4}$-MIBK system, in the south all tantalum and niobium smelting enterprises use $\mathrm{HF}-\mathrm{H}_{2} \mathrm{SO}_{4}$-SEC octyl alcohol system.

The production must ensure tantalum/niobium to be extracted completely, but the impurities not as possible. The main impurities are tungsten, tin, titanium, silicon, iron and antimony in the form of fluorine complex anions. The fluorine complex with titanium or silicon is difficult to be extracted, and the high content can affect split-phase in bad conditions. Tungsten and antimony are the most difficult element to be separated from tantalum/niobium. Tungsten is easily extracted by oxygenated organic solvent. With the increase of hydrofluoric concentration or sulfuric acid concentration, the extraction rate of tungsten increased, but it is not obvious for hydrofluoric. When the concentration of sulfuric acid is more than $4 \mathrm{~mol} / \mathrm{l}$, the extraction rate of tungsten is increased sharply.

Therefore, in order to prevent tungsten into organic phase, acidity must be strictly controlled, especially sulfuric acid. The acidity of sulfuric acid in acid aqueous solution obtained by digestion or the acid washing solution can not exceed 8 mol/l [2]. The experiment and production experience of many years in China show that the extracted order of the main elements in the two major extraction systems is titanium $<$ tungsten $<$ niobium $<$ tantalum. that shows, tungsten is the boundary impurity element separated, and the extraction rate of tungsten is the highest compared with the other impurity elements, the partition coefficient with niobium is the least. Therefore, the extraction-acid washing section is also called the separation unit of tungsten and niobium.

\subsection{Process Principle}

The extraction of tantalum and niobium is a feigned salt extraction. High acid extraction and low acid back extraction is the main characteristic of this extraction. The oxygen extractant generates feigned salt cation with hydrogen ion at high acidity, and then feigned salt cation combines with the complex fluoride anions with tantalum and niobium in the acid aqueous solution into feigned salt which is soluble in organic solvent. While oxygen solvent extracts tantalum and niobium, the total hydrogen ion should be more than $10 \mathrm{~mol} / \mathrm{l}$. When hydrofluoric acid is below $7 \mathrm{wt} \%$, niobium is hydrolyzed into niobium fluoxobate $\left(\mathrm{NbOF}_{5}{ }^{2-}\right.$ or $\left.\mathrm{HNbOF}_{5}\right)$ which is not easy to be extracted into organic solvent, but tantalum remains in tantalum fluoric $\left(\mathrm{TaF}_{7}^{2-}\right.$ or $\left.\mathrm{HTaF}_{7}^{-}\right)$. So niobium can be separated from tantalum for the small difference between niobium an tantalum.

\subsection{Process Flow}

The fractional extraction of tantalum and niobium includes extraction-acid washing section, back extraction niobium extraction tantalum section and back extraction tantalum section, as shown in the attached figure 1. For some low grade refractory ores, an ore pulp extraction process is required before extraction, that is, without filtering the ore is directly extracted after digested, then the organic solvent containing tantalum and niobium is brought into the extraction-acid washing section or acid washing unit

By adjusting the acidity and the tantalum and niobium quantity of the acid aqueous solution by digestion, the extraction rate of tantalum and niobium can be controlled to the largest, the impurities like silicon, iron, manganese, titanium, etc. the least, and the preliminary separation tantalum and niobium from impurities is achieved. The main effect of acid washing is to back extract impurities from organic phase into the aqueous solution. After extraction section the acid washing solution is brought into countercurrent contact with the organic solvent containing many metals to further separate tantalum, niobium from impurities. However the trace tantalum and niobium (mainly niobium) also enter the aqueous solution, thus organic solvent is necessary to extract them again to ensure that the total concentration of tantalum and niobium pentoxide in the residual solution is less than $1 \mathrm{~g} / \mathrm{l}$.

The back extraction niobium section is to separate niobium from tantalum in the organic solvent. The complex fluoric 
with niobium in the organic solvent is destroyed by back extraction solution. The niobium is selectively removed into the aqueous solution, and the trace tantalum is also removed to the aqueous solution. So the fresh organic solvent is necessary to reextract the trace tantalum. Finally pure niobium solution is obtained.
The back extraction tantalum section is to remove completely tantalum to the aqueous solution by pure water, which is essentially the same as that of the back extraction niobium. But the purity tantalum solution obtained is much less acidic than the purity niobium solution.

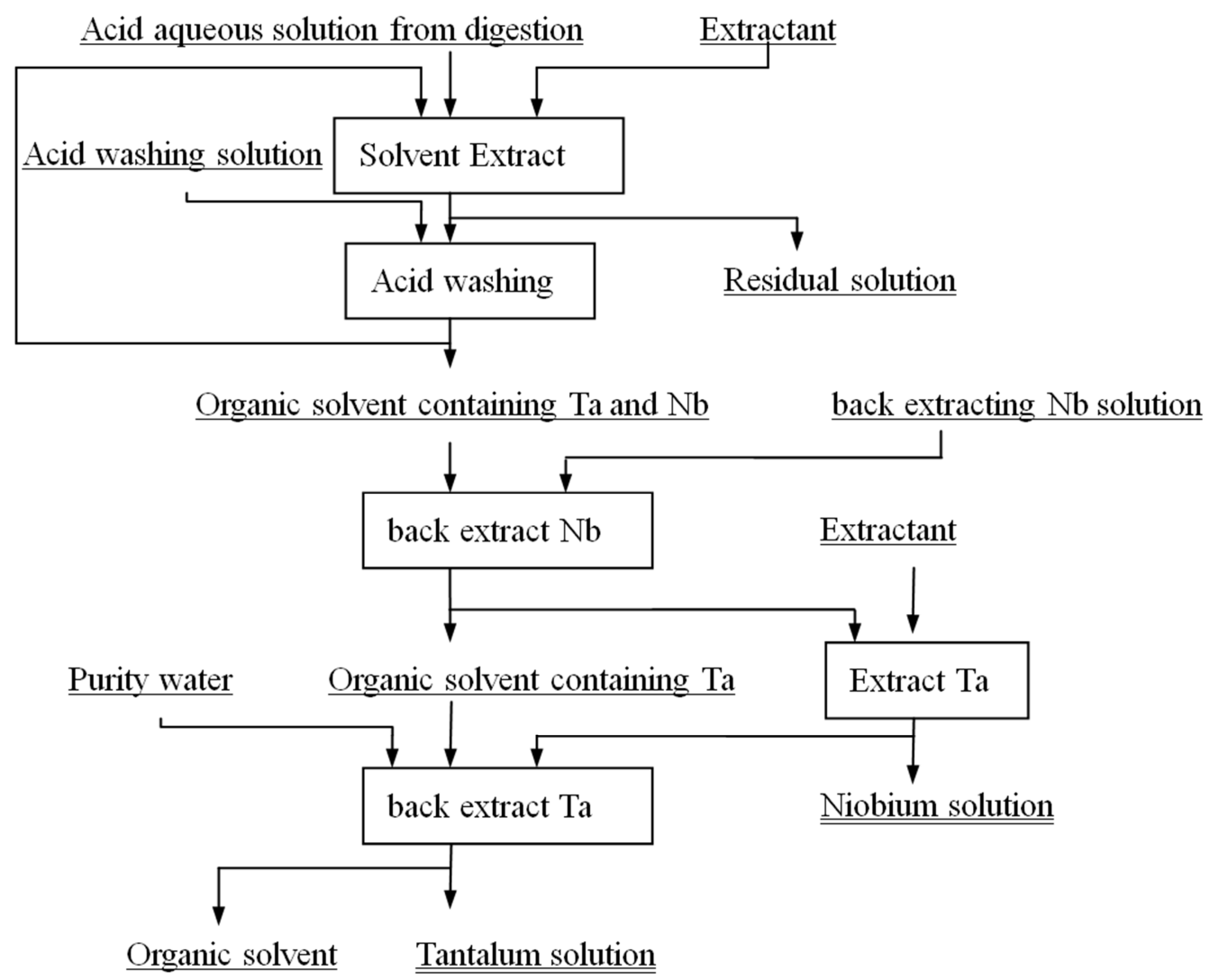

Figure 1. Flow sheet of tantalum and niobium extraction separation.

\subsection{Technological Factors}

\subsubsection{Acidity}

According to the extraction principle, the acidity of aqueous solution is the most important factor affecting the extraction, determines the extraction rate of tantalum/niobium, and the impurity removal efficient and the separation effect of tantalum and niobium. When the hydrofluoric quality concentration is very low $(<7 \%)$, the hydrofluoric concentration is the main factor of affecting the extraction niobium. When the hydrofluoric concentration is more than the quantity required to form $\mathrm{TaF}^{6-}$ and $\mathrm{NbF}^{6-}$ completely, the extraction niobium closely depends on the sulfuric acid quantity.

With the increase of hydrofluoric or sulfuric acid concentration, the extraction rate of niobium increases obviously, the tantalum extraction rate increases little, and the highest point of extraction appears in the extraction curve [2], because hydrofluoric strives to be extracted into the organic solvent and forms the solvent (such as MIBK HF).

When the concentration of hydrofluoric and sulfuric acid are high, the extraction rate of tantalum and niobium are both high, and the separation efficiency of both of them is low, this is the co extraction area of tantalum/niobium. When the concentration of hydrofluoric and sulfuric acid are low, the extraction rate of tantalum is still very high, but the extraction rate of niobium decreases sharply, and the separation efficiency of the both is large, this is the separation area of tantalum and niobium.

The acidity in extraction-acid washing system can be adjusted by controlling the acidity of the aqueous solution and the acid washing solution. The acidity of both of them must be accurate, the flow speed of three phases (organic solvent, acid aqueous solution and acid washing solution) must be kept constant to ensure the extraction balance and stability of the system.

\subsubsection{Stage Efficiency}

The stage efficiency refers to the ratio of the theoretical stages to the actual stages. The stage efficiency of the mixed clarification tank is generally 0.75 to 0.90 . The extraction rate of tantalum/ niobium are increased with the extraction stages increased, but the extraction rate of various impurities will are also increased, which results in poor acid washing effect. The suitable stages must be selected to separate tantalum/niobium 
from impurities. If the acid washing stages are not enough, the impurities may not be eluted to the aqueous solution, which leads to the unqualified products; too much acid washing stages will cause coextraction difficultly, which may lead to the unqualified residual solution and cause the large loss of tantalum/niobium. The stages of extraction and acid washing should be properly matched to ensure the quality of products and the recovery rate of tantalum/ niobium.

The main factors of stage efficiency are the flow speed, rotation rate, size, shape and installation position of the paddle. If the flow speed is too fast, the residence time of the two phases in the mixing chamber is short so that the stage efficiency decreases. If the rotating rate is too low, the mixing effect is low and the two phases can not be fully contacted. While the rotating rate is too high, the solution is dispersed too fine, easy to emulsify, and the lamination is not good.

\subsubsection{Temperature}

When temperature changes, the density difference and viscosity of two phases change, which influences the stratification speed. The viscosity of second octyl alcohol increases with the decrease of temperature, which is disadvantage to two phases mixing and separation. The resistance of liquid transferring is increased so that the emulsion is often accompanied in back extraction process. The indoor temperature difference between winter and summer is large in the south, and the process parameters of secondary octanol system should be adjusted accordingly with seasonal changes. Juan $\mathrm{Xu}$ [3] studied the relationship between the temperature and partition ratio of HF-MIBK extraction system. It was concluded that the reaction of MIBK extraction tantalum was exothermic $(\Delta H=-37.4 \mathrm{~kJ} / \mathrm{mol})$ and low temperature was more suitable.

\subsubsection{Tantalum/Niobium Concentration and Mass Ratio in Acid Aqueous Solution}

With the concentration of tantalum/niobium is increased, the extraction rate decreases, the partition coefficient decreases, and separation coefficient of tantalum and niobium increases. However, in order to increase yields and economic benefits, high concentration is generally used, and low extraction rate is solved by multistage extraction.

When the mass ratio of tantalum and niobium in the acid aqueous solution is large, the tantalum content in both the niobium solution and the organic solvent are high, which is beneficial to the preparation of high-purity tantalum. but it is not conducive to the preparation of high-purity niobium. On the contrary, the acid aqueous solution with small mass ratio of tantalum and niobium are more suitable for producing high-purity niobium, but it is not conducive to the production of high-purity tantalum.

\subsubsection{Extractant}

In the $\mathrm{HF}-\mathrm{H}_{2} \mathrm{SO}_{4}$ - secondary octanol system, the extraction capacity of secondary octanol is decreased for reuse, and this is not obvious in high acid extraction, but it is fatal for extracting tantalum in the low acid, which shows that the tantalum in the niobium solution is not completely extracted.
Especially in the pulp extraction, some fine particles in the pulp are easily adsorbed by the large viscosity organic matter to form the "black slag", which causes the two phases stratification difficult and the mutual entrainment is serious, resulting in the loss of organic phase and tantalum and niobium.

Diluted industrial secondary octanol with kerosene could reduce the viscosity of organic phase and facilitate the separation of two phases. $75 \%$ secondary octanol plus $25 \%$ kerosene was good to titanium/niobium pulp extraction; and also could be well used to extract tantalum in back extraction niobium-extraction tantalum section, but the concentration of back extraction niobium solution was increased [4]. In the back extraction niobium-extract tantalum section the viscosity of the organic phase is not the main factor, and the non diluted secondary octanol should be selected for extracting tantalum [5]. The organic phase for extracting tantalum is often refined, that is, pure water and other detergents are used to remove impurities and restore its extraction capacity.

\subsubsection{Volume Ratio}

Volume ratio is the volume ratio of the organic phase to the aqueous phase. The actual operation is controlled by calculating the inlet and outlet balance flow of the tantalum/niobium in system. The extraction rate of tantalum/niobium increases with the increase of the volume ratio. The flow of acid washing solution increases, and the washing effect is better. However, if the volume ratio is too large, the acidity of the system is too low, and the extraction of tantalum/niobium is not complete. With the increase of the flow of back niobium solution, the recovery rate of niobium increases, but the concentration of niobium solution decreases, and the purity of niobium product decreases.

\section{Precipitation Process}

Precipitation is to add into tantalum (niobium) solution, so that tantalum (niobium) can be converted from liquid to solid, and then pure tantalum (niobium) intermediate product can be obtained by washing. Precipitation and washing are important processes which affect the purity of products. Precipitants should be removed in subsequent processes and do not cause new pollution to products. Industrial precipitating agents are ammonia, ammonia or liquid ammonia, and some people have tested with urea and ammonium carbonate. When high-purity tantalum or niobium product is prepared, precipitant is required to be cleaned before precipitating to ensure its purity.

When the solution $\mathrm{pH}$ is about 8 to 9 in precipitation, precipitation is finished. In the precipitation process with the gradual increase of $\mathrm{pH}$, each impurity metal generates the corresponding hydroxide which enters tantalum (niobium) hydroxide. The initial $\mathrm{pH}$ with forming hydroxide precipitation is related to the valence state and activity (effective ion concentration) of the metal ions. The precipitation of some metal impurity can be removed by adjusting the valence state or controlling the $\mathrm{pH}$ by step precipitation. Weining Zhang and other [6] can accurately 
control the $\mathrm{pH}$ in the neutralization of niobium (tantalum) solution. The antimony hydroxide is precipitated firstly by step precipitation, and the filtrate is then neutralized to the end point. The $\mathrm{pH}$ of step precipitation was 5 to 6 and 9 to 10 respectively, and the content of antimony in niobium hydroxide decreased from $0.03 \mathrm{wt} \%$ to $0.0025 \mathrm{wt} \%$.

Separating antimony by sulfide precipitation method is similar to by hydroxide precipitation, both are based on the difference of $\mathrm{pH}$ between antimony precipitate and niobium precipitate formed. Yaohua Zhang and other [7] used hydrogen sulfide or ammonium sulfide as vulcanizing agent, finally obtained the niobium oxide product with the antimony content less than $0.005 \mathrm{wt} \%$, by adjusting the acidity of niobium solution $\left(\mathrm{Sb}^{3+}\right.$ concentration about $\left.0.5 \mathrm{~g} / \mathrm{l}\right)$ to $1 \sim 2 \mathrm{~mol} / 1$ or $1 \sim 3 \mathrm{~mol} / 1$, heating the niobium solution of high antimony, adding the precipitant and keeping heat for 30 minutes, then added the carrier or electrolyte and keeping heat for one hour, cooling and filtrating.

While precipitating, the residual charge attracts easily impurity ions (such as fluoride ion and sulfate ion) due to the imbalance of ionic charges on the precipitated surface. When the ammonia is constantly added and the reaction is intense, the precipitate particles grow up quickly, the adsorbed impurities with the surface are too late to leave, but the precipitate still continues to grow. The impurity ions are wrapped in the precipitate, causing the product purity to reduce, and this kind of impurity is difficult to be removed. Therefore, the speed of adding ammonia precipitator and the concentration of solution and precipitator should be well controlled, and agitation should be strengthened to prevent excessive local concentration.

The adsorption of impurities on the precipitated surface is exothermic. Increasing the temperature of the solution can reduce adsorption. In addition, the adsorption quantity can be reduced by controlling precipitation conditions to change the morphology of the precipitates, transform them from amorphous to crystalline and reduce the specific surface area.

Jun Liu and other [8] prepared high pure tantalum oxide (niobium) with less than $0.0001 \mathrm{wt} \%$ sulfur content by peroxide treatment with peroxide. The amorphous precipitate was changed into crystalline precipitation and the adsorption capacity of sulfate anion was weakened. The very small amount of sulfate adsorbed was converted to thiosulfate anion $\left(\mathrm{S}_{2} \mathrm{O}_{8}{ }^{2-}\right)$ which leaded to the low temperature digestion of sulfate salt in the subsequent treatment. $0.1 \mathrm{wt} \%-0.5 \mathrm{wt} \%$ peroxide of 5 30liters with per kilo high purity tantalum oxide (niobium) was the best.

The aging time after precipitation also effect the purity of the product. In the aging process, fine particles are gradually dissolved and large particles grow up gradually. With the dissolution of fine particles, the impurities that are adsorbed, retained and contained in the precipitates enter the solution again, which can improve the purity of the precipitates.

\section{Washing Process}

Washing is to dissolve the impurities on the precipitates into the solution and improve the purity of the precipitates. In order to cause new impurities not to be brought, hot dilute ammonia or hot pure water was used for washing solution. The tantalum (niobium) hydrogen oxide is separated from the mother liquid by vacuum filtration or plate frame pressure filtration, and is washed repeatedly with detergent in a vacuum filter tank with a stirring or filter press.

The ammonia in the hot dilute ammonia is mostly in the form of ammonia molecule. The ammonia molecule polarity is very strong. The lone pair electrons and other ions or molecules can form coordination bonds to produce various complex containing probably $\left(\mathrm{NH}_{4}\right)_{2} \mathrm{SO}_{4} \cdot \mathrm{xNH}_{3}, \mathrm{NH}_{4} \mathrm{~F} \cdot \mathrm{xNH}_{3}$ and so on, which are favorable for the elution of impurities. The washing endpoint is usually determined by determining the amount of fluorine in the washing residue. Because the radius of fluorine atom is very small and the electronegativity is very large, it is easier to be adsorbed on the precipitated surface compared with sulfate anion. Therefore, the washing process is also called washing fluorine. The control of fluorine in washing process depends on the purity requirement of tantalum oxide product and the capability of removing fluorine in subsequent calcination process and the ability of wastewater treatment. The most economical and efficient washing method should be selected. At the same time, we should pay attention to the purity of the detergent and the cleanliness of the equipment, so as to avoid potassium, sodium, calcium, iron, silicon and other elements from being brought into the product.

High concentration niobium solution (niobium pentoxide concentration more than $120 \mathrm{~g} / \mathrm{l}$ ) can be pretreated with diluent. Deng Xiaofen and other [9] solved the difficult problem of washing fluorine from niobium hydroxide by this method. In addition, the efficiency of removing fluorine can also be increased and the amount of washing water can be saved by increasing the temperature of washing water and the frequency of mixing.

Dianmo Zheng and other [10] used niobium hydroxide as raw material produced from $\mathrm{HF}-\mathrm{H}_{2} \mathrm{SO}_{4}$-MIBK extraction system, and by countercurrent extraction to remove fluorine. The volume ratio of extractant and water deionized was 1 to 1 , extraction time is 20minutes, washing was 10 times, calcination temperature was $800^{\circ} \mathrm{C}$. Baiquan Jiang and other [11] used the leaching agent to remove fluorine of tantalum hydroxide and prepared high purity tantalum oxide with low fluorine below $6 \mathrm{~g} / \mathrm{m}^{3}$. The optimal process conditions was as below: 1 to 1 volume ratio of leaching agent to water, $10 \mathrm{~min}$ immersion, 6 times washing and 800 degrees centigrade calcination temperature.

To remove the ammonia ion in niobium hydroxide, Qi Wang and other [12] analyzed infrared spectroscopy and made a conclusion that the chemical formula of niobium hydroxide adsorption ammonia is $\mathrm{NbO}_{\mathrm{x}}(\mathrm{OH})_{\mathrm{y}}\left(\mathrm{NH}_{4}\right)_{\mathrm{x}}$. The Ammonia could be exchanged from the surface of the niobium hydroxide by dilute nitric acid or sulfuric acid solution, but sulfate anion was weakly adsorbed on the surface of niobium hydroxide. Kuang Guozhen and other [13] prepared tantalum oxide with less than $0.006 \mathrm{wt} \%$ nitrogen content by washing 
tantalum hydroxide (fluorine content less than $0.15 \mathrm{~g} / \mathrm{l}$ ) with $75 \sim 85^{\circ} \mathrm{C}$ to less than $0.006 \mathrm{wt} \%$ nitrogen content, then putting steam into the distiller and keeping pressure $0.1 \sim 0.5 \mathrm{MPa}$ for 3 6 hours and calcining.

\section{Calcination Process}

Calcination is the process of decomposing tantalum hydroxide (niobium) into oxide and removing volatile substances such as ammonia and fluorine. Calcination can remove most of the fluorine. If appropriate amount of steam was putted into, the effect of removing fluorine was better, because the metal fluoride can be hydrolyzed. The hydrolysis reaction: $\mathrm{MeF}_{2 \mathrm{n}}+\mathrm{nH}_{2} \mathrm{O}=\mathrm{MeO}_{\mathrm{n}}+2 \mathrm{nHF}$. In addition to controlling the calcination temperature, it is necessary to create good ventilation conditions for volatile substances and strictly controll the furnace load quantity and exhaust quantity.

Ningxia non-ferrous metal smelter has studied to remove fluorine process with pyrohydrolytic method. The main point of the process is that the tantalum (niobium) hydrogen oxide is heated to $700 \sim 800^{\circ} \mathrm{C}$, and the purified steam is passed the material. The residual fluorine in the material is taken away in the form of hydrogen fluoride gas, and the fluorine content in the product can be reduced to less than $0.001 \mathrm{wt} \%$ [14]. Wannian $\mathrm{He}$ and other [15] obtained tantalum oxide of fluorine content less than $0.001 \mathrm{wt} \%$. 10 kilo tantalum hydroxide with $0.47 \mathrm{wt} \%$ fluorine content of and 20 kilo with $0.51 \mathrm{wt} \%$ fluorine content respectively were placed in distillation-calcination device, then $90^{\circ} \mathrm{C}$ steam were poured into for 120 minutes by $400 \mathrm{l} / \mathrm{min}$ and $600 \mathrm{l} / \mathrm{min}$ respectively. The fluorine content of tantalum oxide was only $0.036 \mathrm{wt} \%$ and $0.054 \mathrm{wt} \%$ respectively by common calcination.

\section{Research Progresses of Separating Impurity Technology}

The purity of tantalum (niobium) oxide is not dependent on the control of a single process, but is the result of the interaction of various processes. The focus of acid digestionextraction process is the separation niobium from tantalum, and from metal impurities. The precipitation-calcination process is more concerned with non-metallic impurities. The tantalum (niobium) solution produced by the extraction technology contains hydrofluoric acid and sulfuric acid, and the acidity, fluorine and sulfuric content in niobium solution are more than that in tantalum solution because of different back extraction solution. The main task of the precipitation-calcination process is to change the morphology of tantalum (niobium) while removing impurities such as fluorine, sulfuric and precipitants. But this process can also remove metal impurities. In addition, tantalum oxide is more easily removed than niobium oxide, which is determined by the difference in the behavior of tantalum and niobium in the technologic system, for example tantalum is more easily extracted than niobium, and tantalum solution component is relatively simple than the niobium liquid component. In conclusion, the removal of impurity elements should be considered as a whole and every process should be well controlled.

Liang Tang [16] lowered tungsten content in niobium solution produced by $\mathrm{HF}-\mathrm{H}_{2} \mathrm{SO}_{4}$-secondary octanol extraction from low grade ores of tantalum and niobium. The acidity of acid washing section and extraction section was adjusted: the sulfuric acid concentration of the original acid lotion was decreased to $3.5 \mathrm{~mol} / \mathrm{l}$ from $3.65 \mathrm{~mol} / \mathrm{l}$, and the ratio of the original acid washing to loaded organic flow rate was increased to 0.45 from 0.4 . The hydrofluoric acid aqueous solution was added to the extraction section to increase the total hydrofluoric acid concentration in the loaded organic solvent from $2.8-3.5 \mathrm{~mol} / 1$ to $4 \mathrm{~mol} / \mathrm{l}$. The content of tungsten in final niobium oxide was reached below $0.003 \mathrm{wt} \%$ which can meet the requirements of customers.

Conghua tantalum niobium smelter [1] studied and found that the ability to be extracted of antimony is between niobium and tantalum, ranking $\mathrm{Nb}<\mathrm{Sb}<\mathrm{Ta}$. The separation coefficient of tantalum and antimony was between 1.4 and 5.5, and the separation coefficient between antimony and niobium was between 1.2 and 6 , and the two coefficients were almost equal. Therefore, under the same separation conditions, antimony moved on both sides of the back extraction niobiumextraction tantalum section. Antimony was not only in niobium solution but also in the organic solvent containing tantalum, and finally was brought into tantalum oxide and niobium oxide.

HARVEYS and other [18] believed that the antimony in the niobium solution was mainly positive trivalent. When antimony was in a high valence state (positive quadruple or positive five valence), the hydrofluoric acid concentration is adjusted to $3 \sim 10 \mathrm{~mol} / \mathrm{l}$, antimony and only a small fraction of niobium were be extracted into organic solvent. The trivalent antimony in niobium solution was oxidized into pentavalent antimony by strong oxidants such as hydrogen peroxide and sodium peroxide. After MIBK was used for 15 stages extraction (volume ratio 1.5 to 1 of MIBK and niobium solution) the antimony in niobium solution was reduced to 2 $\mathrm{mg} / \mathrm{l}$ from the original $300 \mathrm{mg} / \mathrm{l}$.

The orient tantalum industry [19] studied to reduce the impurity content and improve the quality of niobium oxide. An enhanced acid washing was added after the original acid washing process. The back extraction niobium section was divided to three segments according to the tantalum and Niobium mass ratio of the raw materials, ABF method was used to regenerate extractant.

High acid raffinate produced by high acid extraction is difficult to be treated. For this reason, Juan $\mathrm{Xu}$ [3] studied the separation of niobium and tantalum technology in low concentration hydrofluoric acid-MIBK system. After extracting tantalum from low acid aqueous solution, the remained niobium solution was crystallized by oxalic acid. The optimal conditions of the technology were as follows: hydrofluoric acid concentration $0.5 \mathrm{~mol} / 1$, contact time 5 minutes, extraction volume ratio 5 to 1 , total concentration of tantalum and niobium $60 \mathrm{~g} / \mathrm{l}$, tantalum and niobium mass ratio 
2 to 1 . The single stage extraction efficiency of tantalum and niobium was $96.01 \mathrm{wt} \%$ and $0.48 \mathrm{wt} \%$ respectively, and the separation coefficient of tantalum and niobium is nearly 50000. The impurities as iron, titanium, aluminum and silicon were hardly extracted and were remained in the raffinate when respective content was less than $1 \mathrm{wt} \%$. All trace niobium could be removed by washing organic phase containing tantalum with $3 \mathrm{~mol} / \mathrm{l}$ sulfuric acid for 2 times.

Guozhen Kuang et al.[20] prepared ultra high purity tantalum (niobium) pentoxide product from high purity (99.99\%) tantalum (niobium) oxide by adjusting the concentration of hydrofluoric acid and sulfuric acid, technological parameters and technological conditions, optimizing the extraction separation coefficient, replacing the analytical sulfuric acid in $\mathrm{HF}-\mathrm{H}_{2} \mathrm{SO}_{4}$-secondary octanol system; and purifying the resulting tantalum and niobium solution to remove antimony effectively and other impurities (silicon, iron, tungsten, titanium) a little.

Mingyuan Wan ${ }^{[21]}$ prepared high pure $(99.99 \%)$ tantalum oxide from industrial tantalum solution produced by $\mathrm{HF}-\mathrm{H}_{2} \mathrm{SO}_{4}$-secondary octanol system as raw material. N503+ kerosene + modifier A as extractant were used to extract partly from low acidity solution that $30 \%$ tantalum was extracted into the organic solvent and the raffinate was still as industrial tantalum solution. The resulting organic phase containing tantalum was multistage back extracted with $\mathrm{pH}$ 9-10 dilute ammonia solution in low volume ratio, then was purified and ammonia neutralized, washed with high purity water, dried and calcinated.

Yi Qing $\mathrm{Hu}$ [22] produced qualified high pure tantalum oxide powder by extracting with $20 \% \mathrm{~N} 503+30 \%$ sec octanol $+50 \%$ kerosene $-\mathrm{HF}-\mathrm{H}_{2} \mathrm{SO}_{4}$ system and taking some purifying measures. The purifying measures included that liquid ammonia was purified with pure water, PVC material was used for washer, copper screen mesh was replaced with nylon screen mesh.

\section{Conclusion}

Extraction of tantalum/niobium is the key process to remove metal impurities in products. The main factors affecting the extraction separation are acidity, grade efficiency, temperature, extractant, concentration of tantalum/niobium, mass ratio of tantalum to niobium and volume ratio. In actual operation, the flow of each phase should be adjusted by the actual conditions and kept constant. Tungsten is the boundary element to be separated in the acid washing section. The acidity of sulfuric acid in material solution and acid washing solution should be strictly controlled below $8 \mathrm{~mol} / \mathrm{l}$. The capability of antimony be extracted is between niobium and tantalum. Under the same separation condition, antimony moves on both directions of back extraction niobium-extraction tantalum section and finally enters tantalum oxide and niobium oxide at the same time.

In the process of precipitation and calcination the reagent purity, the equipment material, and the environmental cleanliness must be concerned to avoid the inclusion of impurity elements. The separation of impurities in the precipitation process is influenced by the concentration and the temperature of tantalum (niobium) solution, the precipitant concentration and adding speed, stirring and aging time. Some impurities can be removed by controlling precipitation conditions, adjusting the valence state of impurity elements or controlling $\mathrm{pH}$ step precipitation. The separation impurities is affected by the temperature of calcination furnace, the furnace charging number and the exhaust volume; Fluorine, sulfate, ammonium and other non-metallic impurities were washed to be removed; thermal hydrolysis is good to remove fluorine.

The improvement of the traditional technology can meet the special low requirement of one or several elements in the product, such as fluorine, tungsten, antimony, etc. But the existing state and transformation behavior of various elements in the processes of decomposition, extraction, neutralization and calcination are rarely reported and need to be further studied. To reduce the fluctuation of the traditional technology and accurately control the content of elements, automatic control technic is required. Environmental problems such as slag storage, high acid residue and ammonia wastewater discharge produced by traditional technology need to be solved urgently to realize sustainable development.

\section{References}

[1] Jianshe Han, Yong Zhou, Process and Equipment Progress of Tantalum Niobium Extraction Separation, Rare Metals and Cemented Carbide, 2004, 32 (2), 15-20.

[2] Ming Wu, Tantalum and Niobium Metallurgy Technology, Beijing, 1986.

[3] Juan Xu, Basic Research on Extraction and Separation of Niobium and Tantalum by MIBK in Low Concentration Hydrofluoric Acid System, Beijing Chemical Industrial University, 2010.

[4] Yaohua Zhang, Ming Chen, Effects of Different Concentrations of SEC Octanol on the Extraction and Back Extraction Properties of Tantalum and Niobium, Rare Metal and Cemented Carbide, 1996 (2), 6-9.

[5] Mingyuan Wan, Improvement of Extraction and Separation Process for Low Grade Tantalum and Niobium Minerals, Cemented Carbide, 2002, 19 (1), 29-31.

[6] Weining Zhang, Jing Li, Jun Liu, Technology Study on Removal Metal Impurities Like Ti, Sb, etc. in $\mathrm{Nb}(\mathrm{OH})_{5} / \mathrm{Ta}(\mathrm{OH})_{5}$ by Stepwise Deposition, Ningxia Engineering Technology, 2002, 1 (3), 216-217, 220.

[7] Yaohua Zhang, Ming Chen, Removal Antimony of Niobium Solution from Tantalum and Niobium Extraction by Sulfide Precipitation Method, Rare Metal and Cemented Carbide, 1994 (2), 54-57.

[8] Jun Liu, Wei Wang, Peisheng Zheng, Technology Study on Removal of impurity sulfur from $\mathrm{Ta}_{2} \mathrm{O}_{5} / \mathrm{Nb}_{2} \mathrm{O}_{5}$, Rare Metal Bulletin, 2005 (10), 36-38.

[9] Xiaofen Deng, Caiming Wang, Study on Reducing Fluorine Content in $\mathrm{Nb}_{2} \mathrm{O}_{5}$, Rare Metals and Cemented Carbide, 2005, $33(1), 11-13$ 
[10] Dianmo Zheng, Zhenxi Wang, Quan Shui Qing et al., Study on Preparation Low Fluorine and High Purity Niobium Oxide by Extraction, Journal of Nanchang University (Engineering Edition), 2005, 27 (2), 71-73.

[11] Baiquan Jiang, Qingrong Huang, Zheng Dianmo, et al. Study on Preparation of Low Fluorine and High Purity Tantalum Oxide by Solvent Extraction, Journal of Nanchang University (Engineering Edition), 2008, 30 (2), 120-122.

[12] Qi Wang, Heng Jiang, Hong Gong, et al. Infrared Spectroscopic Analysis of Ammonia Absorbed on Surface of Niobium Hydroxide Synthesized by Hydrofluoric Acid Method, Analysis Instrument, 2011 (3), 28-32.

[13] Guozhen Kuang, Jinhua Song, Guoxiang Zhang, et al., A Low Nitrogen Tantalum Oxide and Its Preparation Method, China, CN 201410528130, 2015, 1, 28.

[14] Jilin He, Zongguo Zhang, Zhongting Xu, China Tantalum Niobium Hydrometallurgy, Rare Metal Materials and Engineering, 1998, 27 (1), 9-14.

[15] WanNian He, Sihao He, Xiangdong Wang, et al., Preparation Methods of High Purity Tantalum and Niobium Pentoxide Free Fluorine, Xinjiang nonferrous metals, 1998 (2), 36-42.

[16] Liang Tang, Discussion on the technology of removing impurity elements tungsten in the extraction process of Preparing industrial niobium oxide, Industrial Science and Technology Expo, 2013 (32), 407-407.

[17] Jihua Li, Jinzhong Hao, Study on Extraction of Tantalum and Niobium from High Tungsten Niobium Ore by Hydrometallurgy, Rare Metal, 1989 (6), 510-513.

[18] HARVEY Stewart, Newtown Square, C. Edward Mosheim etc., Process for separating antimony from columbium solutions, AU4518570, 1985, 5, 21.

[19] Jilin He, Zongguo Zhang, ZhenDa Lu, etc., Progress in nonferrous metals (vol 5), rare metals and precious metals (tantalum, niobium), Beijing, China Nonferrous Metal Industry Association, 2006.

[20] Guozhen Kuang, Huogen Zhou, Hao Zhang, et al., Preparation of Super High Pure Tantalum Oxide and Ultra High Pure Niobium Oxide by Sec Octyl Alcohol- $-\mathrm{HF}-\mathrm{H}_{2} \mathrm{SO}_{4}$ System, Rare Metal and Cemented Carbide, 2005, 33 (2), 1-3, 8.

[21] Mingyuan Wan, Preparation High Purity Tantalum Oxide by N503 Two-time Extraction Method, Rare Metal and Cemented Carbide, 2002, 30 (2), 19-22.

[22] Yiqing Hu, Preparation of High Purity Tantalum Oxide, Rare Metal and Cemented Carbide, 1994 (1), 9-13. 